\title{
Beyond fish eDNA metabarcoding: Field replicates disproportionately improve the detection of stream-associated vertebrate species
}

\author{
Till-Hendrik Macher ${ }^{\ddagger}$, Robin Schütz ${ }^{\ddagger}$, Jens Arle§, Arne Beermann, Jan Koschorreck§, Florian Leese $\AA^{\ddagger}$ \\ ‡ University of Duisburg-Essen, Essen, Germany \\ $\S$ German Environmental Agency, Dessau, Germany
}

Corresponding author: Robin Schütz (robin.schuetz@rub.de)

Received: 22 Feb 2021 | Published: 04 Mar 2021

Citation: Macher T-H, Schütz R, Arle J, Beermann A, Koschorreck J, Leese F (2021) Beyond fish eDNA metabarcoding: Field replicates disproportionately improve the detection of stream-associated vertebrate species. ARPHA Conference Abstracts 4: e64815. https://doi.org/10.3897/aca.4.e64815

\begin{abstract}
Fast, reliable, and comprehensive biodiversity monitoring data are needed for environmental decision making and management. Recent work on fish environmental DNA (eDNA) metabarcoding shows that aquatic diversity can be captured fast, reliably, and noninvasively at moderate costs. Because freshwater ecosystems act as sinks in the landscape, they also collect traces of terrestrial species via surface runoff or when specimens come into direct contact with water (e.g., for drinking purposes). Thus, fish eDNA metabarcoding data can provide information on fish but also on other, even terrestrial vertebrate species that live in riparian habitats. This data become available and may offer a much more comprehensive approach for assessing vertebrate diversity at no additional costs. Studies on how the sampling strategy affects species detection especially of stream-associated communities, however, are scarce. We therefore performed an analysis on the effects of biological replication on both fish as well as (semi-)terrestrial species detection. Along a 2-km stretch of the river Mulde (Germany), we collected $181 \mathrm{~L}$ water samples and analyzed the relation of detected species richness and quantity of biological replicates taken. We detected 58 vertebrate species, of which 25 were fish and lamprey, 18 mammals, and 15 birds, which account for $50 \%, 24 \%$ and $7 \%$ of all native species to the German federal state of Saxony-Anhalt. However, while increasing the
\end{abstract}


number of biological replicates resulted in only $25 \%$ more detected fish and lamprey species, mammal and bird species richness increased disproportionately by $69 \%$ and 84 $\%$, respectively. Contrary, PCR replicates showed little stochasticity. We thus emphasize to increase the number of biological replicates when the aim is to improve general species detections. This holds especially true, when the focus is on rare aquatic taxa or on (semi-)terrestrial species, the so-called 'bycatch'. As a clear advantage, this information can be obtained without any additional sampling or laboratory effort when the sampling strategy regarding biological replication is chosen carefully. With the consideration of frequent eDNA metabarcoding as part of national biomonitoring programs, the additional information provided by the bycatch can be used to further investigate the state of the environment and its biodiversity on a much broader scale.

\section{Keywords}

environmental DNA, metabarcoding, vertebrates, biological replication, fish, mammals, birds, sampling strategies, bycatch

\section{Presenting author}

Robin Schütz

\section{Presented at}

1st DNAQUA International Conference (March 9-11, 2021) 